\title{
Toward the identification and the targeting of key players of the mitochondrial unfolded protein response $\left(\mathrm{UPR}^{\mathrm{mt}}\right)$ in cancer
}

\author{
Doris Germain ${ }^{1}$ (D)
}

Received: 3 May 2017 / Accepted: 11 May 2017 / Published online: 29 May 2017

(C) Springer Science+Business Media New York 2017

Keywords Mitochondrial unfolded protein response · $\mathrm{UPR}^{\mathrm{mt}} \cdot \mathrm{ClpP} \cdot \mathrm{ClpXP} \cdot \mathrm{hsp} 60 \cdot \mathrm{Chop} \cdot$ Sirtuin · SIRT3 · FOXO3A · Manganese dismutase $\cdot$ SOD2 - Estrogen receptor alpha $\cdot \mathrm{ER} \alpha \cdot$ Mitophagy

\section{Introductory comment}

The mitochondrial UPR (UPR ${ }^{\mathrm{mt}}$ ) is rapidly gaining attention. While most studies on the UPR ${ }^{\mathrm{mt}}$ have focused on its role in aging, emerging studies suggest an important role of the UPR ${ }^{\mathrm{mt}}$ in cancer. Further, several of the players of the UPR ${ }^{\mathrm{mt}}$ in mammalian cells have well reported roles in the maintenance of the organelle. The goal of this special issue is to emphasize aspects of the $\mathrm{UPR}^{\mathrm{mt}}$ that have been overlooked in the current literature, describe the role of specific players of the UPR ${ }^{\mathrm{mt}}$ in the biology of the mitochondria and highlight the intriguing possibility that targeting the UPR ${ }^{\mathrm{mt}}$ in cancer may be already within reach. I want to thank all authors for the contributions and a special thank to Dr. Nick Hoogenraad who has pioneered the work on the $\mathrm{UPR}^{\mathrm{mt}}$. This special issue begins with the review of the discovery of the original arm of the $\mathrm{UPR}^{\mathrm{mt}}$, regulated by the transcription factor CHOP leading to the activation of hsp60 and the protease ClpP. This short review is than followed by the description of more recent work implicating ClpP in cancer.

The description of the CHOP axis is than followed by the description of the discovery by our group of two additional axes regulated by the estrogen receptor and SIRT/FOXO3A respectively. The subsequent reviews focus on the connection between the estrogen receptor and the regulation of mitochondrial biogenesis and reviews on SIRT3 and SOD2 in the regulation of the mitochondria. This special edition also includes the description of recent work on the role $\mathrm{FOXO} \mathrm{A}$ in cancer and stem cells and describes the critical role of FOXO3A in nuclear-mitochondrial communication.

The picture that emerges is that the UPR ${ }^{\mathrm{mt}}$ is a complex transcriptional program regulated by several transcription factors to coordinate an extensive response that affects multiple mitochondrial functions including chaperones and proteases but also mitophagy, anti-oxidant, oxidative phosphorylation and mitochondrial biogenesis.

Clearly, the elucidation of this pathway is only beginning and promises to reveal many new aspects in the future with implications in cancer but also several mitochondrial diseases. Finally, as drugs targeting some of the players of the UPR ${ }^{\mathrm{mt}}$ identified so far already exist, the possibility of targeting this pathway appears to be within reach and represents an exiting novel avenue of research.

Doris Germain, PhD

Doris Germain

doris.germain@mssm.edu

Icahn School of Medicine at Mount Sinai, Department of Medicine, Division of Hematology/Oncology, Tisch Cancer Institute, New York, NY 10029, USA 